\title{
ROUTING AND HANDOVER ISSUES OF RSVP MESSAGES IN MOBILE IP ENVIRONMENT
}

\author{
I. Mathes and T. Dagiuklas \\ Advanced Communications Technologies \\ Development Programmes Department \\ INTRACOM SA \\ Peania Attica \\ Athens, GREECE \\ Tel: ++3016690368 \\ Fax: ++3016860312 \\ Email: \{imath,ntan\}@intracom.gr
}

\section{ABSTRACT}

This paper addresses the problems of interworking of RSVP and IP in order to support QoS in hosts within a mobile environment. Two important issues are discussed. The first regards routing of RSVP messages combined with Mobile IP (both IPv4 and IPv6) and the second concerns the impact of handover in the operation of RSVP. The problems associated with each issue are discussed for both Mobile IPv4 and IPv6 and possible solutions have been proposed.

\section{INTRODUCTION}

Recent advances in the mobile computing, personal wireless communications and the Internet have led to the explosion of applications (e.g. WWW, multimedia, audio and video) to the mobile user [1]. There are three reasons, which has led to this explosion. The first regards the latest advances in the increasing computing power in the PCs capable to support multimedia applications. The second concerns the evolution towards Universal Mobile Telecommunication System (UMTS). Finally, the Internet has experienced in the last decade a great explosion due to the innovation of new multimedia services (e.g. WWW, Internet Telephony) containing video and audio elements.

Originally, the Internet had been designed to provide "best effort" service to IP datagrams with no guarantees in loss and delay. However, the provision of multimedia applications must use methods to provide Quality of Service (QoS) guarantees to services such as video and audio. One of the methods proposed to provide QoS in the Internet, is the use of Resource 
Reservation (RSVP) protocol in the routers along the path from the sender to the receiver [4], [19].

The provision of QoS in mobile environment is more complicated due to handover. Handover occurs when either the host moves from one location to another or when the radio resources gets exhausted to the current cell (access point) and therefore the host is handed over a neighboring cell. Mobile IP has taken into account the fact that a host may move around. On the other hand, RSVP considers that the users are always attached to the same access point in the network. There are two important aspects of RSVP interworking with the Mobile IP. The first considers the routing of RSVP packets that may follow different paths from those of the IP datagrams. As an effect, reservation may take place at different path, which may lead to the reduction of the service to the "best effort" category. The second regards the reservation of resources to a new location area when handover occurs.

This paper is organised as follows. Section 2 presents an outline of RSVP. Section 3 and 4 describe the routing problems of RSVP combined with Mobile IPv4 and Mobile IPv6 respectively and proposed solutions are presented. Section 5 discusses the impact of handover in the RSVP and solutions are proposed for both Mobile IPv4 and IPv6. Finally, conclusions are given in section 6 .

\section{RSVP OUTLINE}

RSVP is a setup protocol designed for integrated services. It is used by a host to request specific QoS from the network for a particular flow [4], [6], [19]. RSVP is also employed by routers to deliver QoS requests to all nodes along the path(s) of the flows, establish and maintain states to provide the requested service. RSVP is not a transport or routing protocol. Routing is handled by current routing protocols and transport of data is carried out by protocols like TCP, UDP, RTP and ST2. It operates over the IP layer and employs soft state and refresh policy, so that the generated states in intermediate routers are timed out, unless they are refreshed [4], [6], [19].

The two basic message types of RSVP are PATH and RESV. PATH messages are sent by the transmitters towards the receivers, as illustrated in Figure 1 [7]. They are routed on end-to-end basis as normal data and establish "PATH states" in the intermediate routers. PATH states include information such as the sender of the message, the IP address of previous hop and the requested QoS. Intermediate routers modify PATH messages to describe the service they can provide and forward it to the next hop. On the reception of the PATH message, the receiver generates the appropriate RESV message, which is sent to the previous node using the previous hop 
field of the PATH state. The RESV messages follow exactly the same route as the PATH ones. On the reception of the RESV messages, the routers reserve the appropriate resources and transmit a RESV message towards the node sending the PATH message.

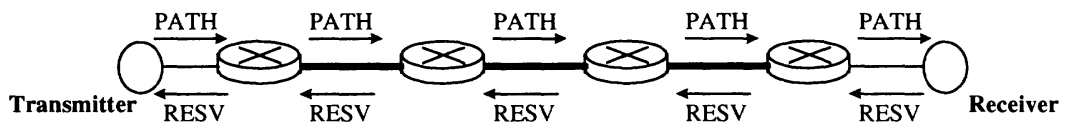

Figure 1: RSVP set-up operation

\section{ROUTING of RSVP MESSAGES}

\section{Routing of RSVP with Mobile IPv4}

In standard Mobile IPv4, all data destined to a Mobile Node (MN) from a Correspondent Node $(\mathrm{CN})$ are routed through the MN's Home Agent (HA) [15], [16]. The CN sends the packets using the MN's home address (ha) as destination. IP packets arriving at the HA, are encapsulated in another IP datagram where the MN's care of address (coa) is used as destination. IP datagrams are delivered to the MN's Foreign Agent (FA) or directly to the $\mathrm{MN}$ if it owns a collocated coa (this is an temporary address assigned to the $\mathrm{MN}$ itself) [15], [16]. A de-capsulation procedure follows prior to sending packets to MN's IP processing module. Transportation of data within the IP tunnel is carried out transparently, so that intermediate routers do not notice the payload of the inner IP datagrams. Routing of IP packets through the HA occurs only in the case when the $\mathrm{CN}$ is a transmitter, since in the reverse direction packets are sent directly to the $\mathrm{CN}$ without the need for tunneling.

The same procedure is applied to the RSVP PATH and RESV messages, which are sent from the $\mathrm{CN}$ towards the $\mathrm{MN}$, as illustrated in Figure 2. In the reverse direction, PATH messages are routed directly to the $\mathrm{CN}$ (if routing decisions are based on the destination address and not the source one), without passing through the tunnel. RESV messages also avoid tunneling since they are routed using the previous hop value kept in the "PATH state" of each router traversed by the PATH messages. 


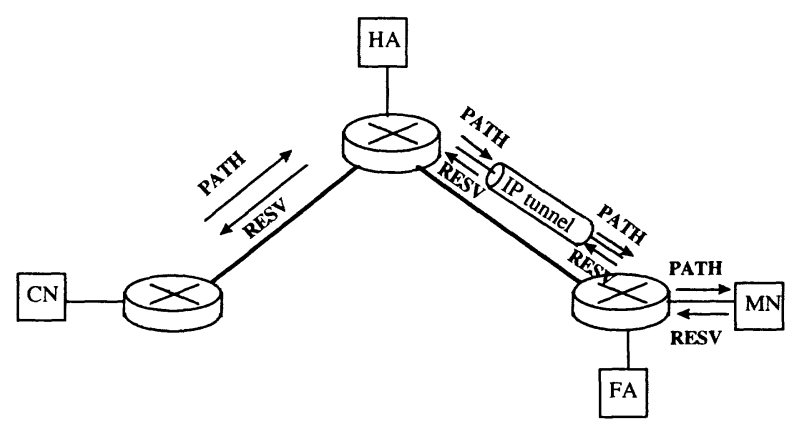

Figure 2: RSVP messages routing in combination with Mobile IPv4.

When the $\mathrm{CN}$ transmits a RSVP PATH message towards the $\mathrm{MN}$, it uses MN's ha as the destination address. As an effect, PATH messages reach the HA where they will be IP encapsulated and are sent to MN using its coa. However, the outer header of the IP tunneled packet will not carry a notification indicating a RSVP message, which must be processed by the intermediate routers. Normally, a Router-Alert option must be carried and the protocol id must be set to 46 indicating a RSVP message. All intermediate routers in the tunnel, until the one at the exit will discard the PATH message. In the same way, the routers within the tunnel will neglect the RESV messages traveling in the opposite direction and no reservation will be made inside the tunnel. Additionally, even if it is possible to reserve resources inside the tunnel, it would be difficult to determine whether the tunneled packets must be handled in a different way or not. This is due to the fact that current IP-in-IP encapsulation scheme moves all distinguishing information to the inner IP header adding only a standard IP header as the external wrapper.

A solution to the tunnelling problem has been proposed by IETF [18]. The entry router (Rentry) of a tunnel recognizes the end to end RSVP PATH [16], [18]. Subsequently, a tunnel PATH message is sent from Rentry towards the exit router of the tunnel (Rexit). This message is not IP encapsulated and the intermediate routers inside the tunnel process it. When this message is received at Rexit, it is processed and deleted. The original end to end PATH message is encapsulated at Rentry and is sent to Rexit. This PATH message carries a new RSVP object. The purpose of this object is to associate the end to end session with the relevant tunnel session. Encapsulated end-to-end PATH messages are decapsulated at Rexit. Prior to forwarding the message to the next hop along the path to the destination, Rexit records the association of the tunnel session with that of the end-to-end 
session, and sets the previous hop field of the end-to-end session to Rentry. At the reverse direction when an end to end RSVP RESV message reaches Rexit, it triggers a tunnel RESV message from Rexit to Reentry, which makes reservations at all intermediate routers inside the tunnel. The end to end RESV message is encapsulated at Rexit and forwarded upstream to Rentry, where it is de-capsulated and forwarded to the next hop. When data packets arrive at Rentry, if there is no resource reservation for them, then they are normally IP encapsulated and forwarded into the tunnel. Otherwise, the encapsulation procedure adds a UDP header within the IP outer header.

We propose an alternative solution to bypass the tunneling problem. In this approach, the intermediate routers in the tunnel process the encapsulated IP datagrams in order to identify RSVP messages, as well as the IP data flows. Such a scheme is illustrated in Figure 3. The RSVP PATH messages travelling in the tunnel are encapsulated into two IP datagrams. When a packet reaches an intermediate router, the router must strip out both the outer and inner IP headers before submitting the PATH message to the RSVP module. In order to strip out the outer header, this message must carry a Router-Alert indication and the Protocol ID field must indicate the transportation of RSVP message within the IP datagrams (a new protocol ID number has to be defined). In order to strip out the inner IP header, a RouterAlert option must be carried in the header and the protocol ID is set to 46 . RESV messages travelling in the reverse direction will not be encapsulated so there is no need for special treatment. Additionally, the correct classification of encapsulated IP datagrams inside the tunnel requires processing based on the inner and not on the outer IP header. By employing a protocol ID to identify IP encapsulation in the outer IP header, intermediate routers can be notified to classify incoming IP packets, using the inner IP header. Then the classification would be correct and match the reserved resources.

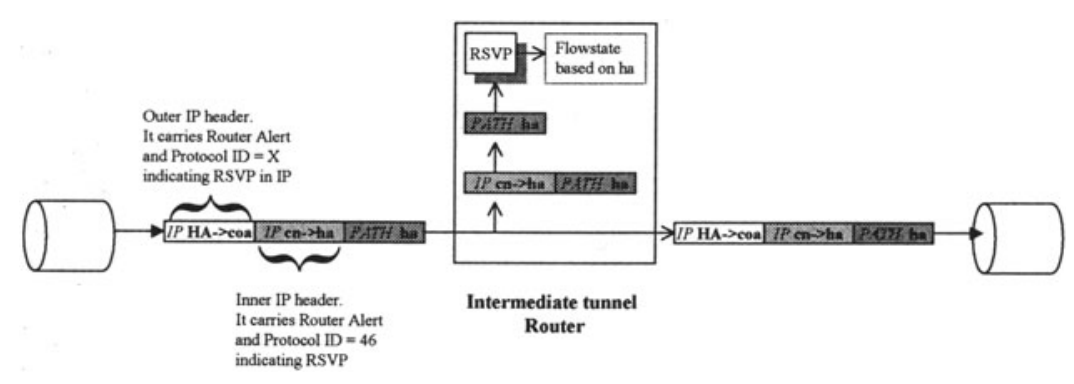

Figure 3: Proposed RSVP Tunneling using Mobile IPv4 


\section{Routing of RSVP Using Mobile IPv6}

In Mobile IPv6, a MN can send binding updates to inform CNs of its current coa [12], [14], [16]. Binding updates may be sent after a binding request has arrived from a $\mathrm{CN}$. CNs may respond binding acknowledgments to notify MNs for the receipt of a binding update message. When a $\mathrm{CN}$ wants to send an IP packet to a MN, a look up of the binding cache is generated prior to the formulation of the IP header. If there is a relevant entry, then MN's coa is obtained from the binding cache and is used as the destination address. MN's ha is placed in an IPv6 Routing Extension Header. It must be emphasised that, by default, in IPv6 all coa are collocated and the employment of FAs is unnecessary [12], [14]. Once IP packets reach the MN, the IPv6 Routing Header will be processed, the ha will be identified as the ultimate destination and the IP datagram will be delivered to the upper layers.

The employment of binding caches alters considerably the routing of IP datagrams, which are sent from the CN towards the MN [12], [14], [16]. IP datagrams are no longer routed through the ha. Instead they follow the optimal route directly to MN's coa. When the CN does not know MN's coa, the MN's ha will be used. As an effect, IP datagrams are sent through the $\mathrm{HA}$ to the MN. In this case, when the MN receives the first tunneled IP packets, it can understand that the $\mathrm{CN}$ does not know its coa and may send a binding update to the $\mathrm{CN}$. After receiving the binding update, the $\mathrm{CN}$ can send IP packets to the MN through the optimal route. The same modifications must be considered for the routing of the RSVP messages, when Mobile IPv6 is employed. Routing RSVP messages using Mobile IPv6, two different cases have been studied. In the first, $\mathrm{CN}$ does know MN's coa whereas in the second case CN knows MN's coa. The problems and possible solutions associated with each case are examined in the following sections.

\section{Routing of RSVP Messages when the CN does not know MN's coa.}

When the CN sets up a RSVP session in the route to a MN, it will transmit an initial PATH message to the MN. The RSVP module in the CN operates over the IPv6 module and the associated binding cache. So the $\mathrm{CN}$ will employ MN's ha when constructing the outgoing PATH message. Additionally, MN's ha will be used as the destination address in the IP datagrams carrying the PATH message, since there is no relevant entry in the binding cache. Therefore, the PATH message will be routed through MN's HA making no reservation inside the IP tunnel.

When the PATH message reaches the MN, it will understand that the $\mathrm{CN}$ does not have an entry in its binding cache associating its ha with the corresponding coa. In this case, a binding update may be sent to the $\mathrm{CN}$. After receiving the binding update, the $\mathrm{CN}$ is able to send IP datagrams to 
the MN through the optimal route, bypassing the HA. However, some IP datagrams may already have been transmitted from the $\mathrm{CN}$. Additionally, the $\mathrm{CN}$ must send a new PATH message to reserve resources through the optimal route. We propose two solutions to alleviate this problem:

- In the first approach, the MN does not send a RESV message as a reply to the initial received PATH message. Instead, it sends a binding update message towards the $\mathrm{CN}$. When the binding update message arrives at the $\mathrm{CN}$, its corresponding RSVP module is notified of the MN address association. However, this solution requires modifications on the operation of RSVP and binding updates, both at the MN and the CN.

- Another solution is to allow the HA to recognise the incoming (from the $\mathrm{CN}$ ) RSVP message and instead of tunneling it, a binding update is sent towards $\mathrm{CN}$. Then, the $\mathrm{CN}$ can initiate a new RSVP session directly to MN. This approach requires modifications in the Mobile IP operating at the HA (a binding update is sent instead of tunneling the received PATH message) and also alterations at the binding update and RSVP operation at the CN (the binding update notifies the RSVP module of the coa-ha association).

\section{Routing of RSVP Messages when the CN knows MN's coa.}

The RSVP PATH message that is sent by the RSVP module of the $\mathrm{MN}$ towards the $\mathrm{CN}$, contains the MN's ha. This message is delivered to the IPv6 module for transmission assuming that an entry exists in the binding cache associating MN's ha and coa. The IP datagrams include MN's coa as the destination address. As an effect, the PATH message is routed to MN through the optimal route without traversing MN's HA. However, since the RSVP PATH message within the IP datagrams contain MN's ha, flowstates at routers will be based on the ha (as illustrated in Figure 4). The IP datagrams requiring a certain $\mathrm{QoS}$, are identified by the session spec (the triple destination address, protocol id and optional destination port) and filter spec (sender id). IP datagrams, though, transmitted by the CN use MN's coa as destination address. $\mathrm{CN}$ assumes that an entry exists in the binding cache associating MN's ha and coa. Although, QoS reservations may exist at the routers, IP datagrams will not be identified as the flow to obtain the reserved QoS since they include MN's coa. 


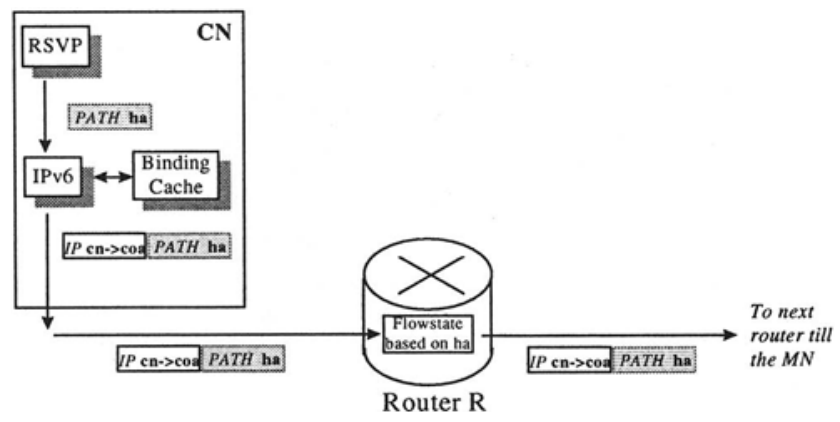

Figure 4: Transmission of PATH message from $\mathrm{CN}$ towards the MN using Mobile IPv6

We propose three solutions in order to resolve the aforementioned problem:

- The first one regards the employment of MN's coa instead of its ha in the RSVP PATH message which is sent from the $\mathrm{CN}$ towards the MN. Then in the intermediate routers, "path states" and reservations must be assigned according to the MN's coa and are compliant with the destination address employed in IP datagrams. This solution can be implemented by modifying the RSVP module so that when the PATH messages are created, the binding cache is accessed to retrieve MN's coa. As an effect, both Mobile IPv6 and RSVP must be modified. Mobile IP must provide a method for enabling RSVP to retrieve binding cache information and RSVP module must access the binding cache prior to the formulation of the PATH message. Another alternative option of including MN's coa in the PATH message, regards the modification of Mobile IPv6 module to recognize the RSVP PATH message. In this case, MN's ha is swapped with MN's coa in the PATH message. This method does not impose modifications in the RSVP module but it requires extensions in Mobile IPv6 to parse all RSVP messages and swap the addresses. This extension may slow down considerably the operation of the Mobile IPv6 module. It must be emphasized that both approaches require modifications at the $M N$ when creating the RSVP RESV message.

- A second solution necessitates the employment of binding caches at the routers. When IP datagrams arrive from the CN using MN's coa as destination address, it is swapped with MN's ha prior to transferring it to the router's decision whether the data belong to a flow or not. As an effect, flow classification is based on MN's ha which is compliant with the employment of ha when the initial QoS reservation at the router is carried out. One method to keep binding caches at the intermediate 
routers, is to process the binding update messages at every visited router along the path from the $\mathrm{CN}$ towards the $\mathrm{MN}$. This method requires modifications at the Mobile IPv6 software at the routers. However, the main disadvantage of this approach regards its scalability when the dimension of the caches gets large. Another approach to update binding caches, is to process the outer IP header containing the PATH messages, which includes both MN's coa and ha. This method exhibits the same scalability drawback as the previous one.

- A third solution is to make QoS reservations at the routers based on both MN's coa and its ha. In order to achieve this, the outer header of the IP datagrams carrying the RSVP PATH message must be provided to the RSVP module residing at the routers. Then the RSVP module will be informed of both MN's coa and ha and a path state is created according to MN's coa. However, the header of the IP datagram including the RSVP RESV message, which is transmitted in the reverse direction, will not contain both addresses (since it is routed hop by hop). Only MN's ha will be included in the inner RESV message. Thus, the RSVP module must maintain a mapping between MN's coa and ha (something like the binding cache), to create correctly the QoS reservation when the RESV message arrives.

\section{RSVP and Handover}

In a wireless/mobile IP environment, Mobile IP can be used in order to handle mobility and RSVP can be employed (assuming an integrated service model) to handle the reservation resources part. As a mobile host moves from one Access Point (AP) to another, mobility and resource reservation procedures must be employed in order to register the $\mathrm{MN}$ with the new AP [10], [13]. If the applications running at the MN are adaptive, a re-negotiation of the QoS that was originally allocated can be made, which could even degrade the service offered to "best effort" [2]. However some connections may not accept QoS re-negotiation. These connections will either be dropped or an alternative method should be employed to assure the existence of enough resources in the new AP [2], [8], [9]. In a Mobile IP based network, RSVP module must manage the reservations in the new path when the MH experiences handover. This necessitates possible extensions of the RSVP and interactions of RSVP module with Mobile IP to operate while $\mathrm{MH}$ experiences handover. These issues will be addressed in the following sections both for Mobile IP v4 and v6. 


\section{Handover and RSVP in Mobile IPv4}

In standard Mobile IP (Mobile IPv4, or Mobile IPv6 without using binding cache option), all IP datagrams from a $\mathrm{CN}$ to a $\mathrm{MN}$ are routed through the MN's HA. The HA tunnels the arriving datagrams either to the MN's FA or directly to the MN (if a co-located address scheme is employed). In this scenario, resource reservation is carried out in the routers from the $\mathrm{CN}$ to the FA through the HA. No reservation exists inside the tunnel, unless specific extensions for RSVP are employed. When the MN hands over from a FA1 to a FA2, the path from $\mathrm{CN}$ to HA remains the same and new resource reservation is necessary only at FA2. The following steps occur while handover is performed:

1. The MN moves from FA1 to FA2.

2. The MN listens to the FA2 advertisements and registers with it

3. The HA is notified for the MN's new position.

4. Once "refresh timeout" period is expired, the HA sends a PATH message towards the MN. These messages will be transmitted through a new IP tunnel between the $\mathrm{CN}$ and the FA2.

5. Once the PATH message arrives at FA2, a new PATH state is created which is forwarded towards the MN.

6. In the next step, the MN sends the appropriate RESV message to FA2, which completes the resource reservation at FA2.

7. Resource reservation at FA1 will be deactivated at the expiration of the "cleanup timeout" interval at FA1.

Figure 5 illustrates the handover procedure regarding the RSVP operation with standard Mobile IP (v4).

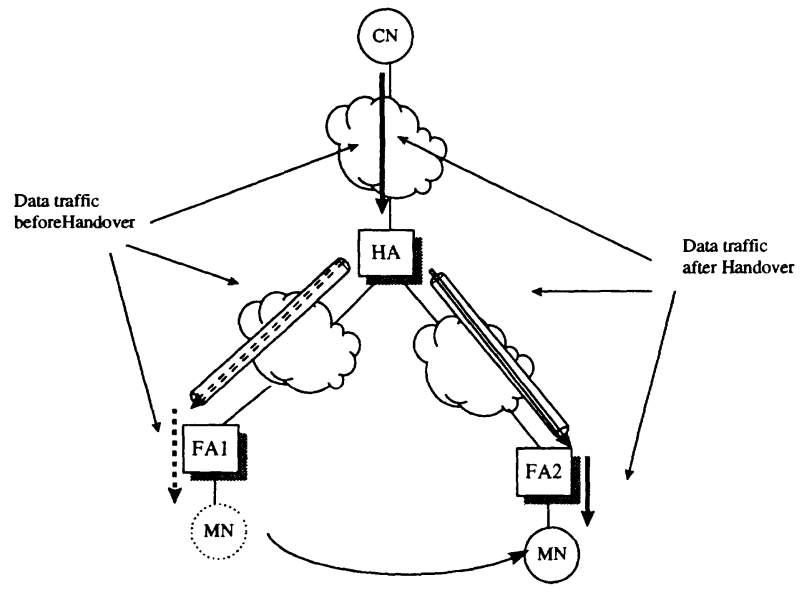

Figure 5: The handover procedure in combination with Mobile IP v4 and RSVP 
The drawback of the above procedure is that the new resource reservation at FA2 is performed only after the expiration of the "refresh timeout" period at the HA. As an effect, a limited number of datagrams from the $\mathrm{CN}$ to the $\mathrm{MN}$ will traverse the path towards MN receiving "best effort" QoS at FA2. Additionally, it must be noted that steps 2-6 are based on "best effort" transport, which extends also the duration of handover/resource reservation procedure.

Mobile RSVP (MRSVP) has been proposed in order to solve the aforementioned problem of the RSVP operation during handover [17]. The main idea of MRSVP is that the MN makes in advance resource reservations in all possible cells it may visit. In order to achieve this, the MN must be capable of a mobility specification (Mspec), which is a set of locations, the MN may visit. However, the main disadvantage of MRSVP is the overreservation of resources, which are wasted.

We propose an alternative scheme, which alleviates the overreservation drawback of MRSVP. In this approach, the initiation of the new resource reservation at FA2 is employed by forcing the HA to send the PATH message towards FA2 immediately after being notified about MN's new position (coa2). Additionally, a PATH Tear message can be also sent towards the FA1 in order to tear down the old reservation at FA1 and release the resources dedicated to this flow. In order to implement this solution, the RSVP module at the HA must interact with Mobile IP. Once Mobile IP informs the HA about the movement of the MN towards a new AP, it triggers the RSVP module to modify the coa address of the MN. By employing this procedure, the HA is enabled to send the PATH message before the expiration of the relevant "refresh timer". However, this method requires modifications both in the RSVP module and Mobile IP at the HA.

\section{Handover and RSVP in Mobile IPv6}

The employment of binding caches in Mobile IPv6 allows the $\mathrm{CN}$ to communicate directly with the MN without routing its traffic through the HA. This option in Mobile IPv6 necessitates the employment of the optimum route from the $\mathrm{CN}$ to $\mathrm{MN}$ and permits the reservation of resources throughout the entire path (there is no tunnel present). When a MN moves from a FA1 to a FA2, a new resource reservation is carried out from the CN to the new location of the MN. The following procedures are carried while handover is performed:

1. The MN listens to the FA2 advertisements and registers with it

2. The HA is notified for the MN's new position (coa2). 
3. The $\mathrm{MN}$ sends binding updates to the $\mathrm{CN}$ to notify about its current location (coa2).

4. The $\mathrm{CN}$ sends a new PATH message to trigger a new resource reservation procedure along the new path.

5. The MN will transmit back a RESV message as a response to the received PATH message, completing the resource reservation procedure.

6 . The connection can now proceed in the new cell.

Figure 6 illustrates the handover procedure regarding the RSVP operation with Mobile IPv6.

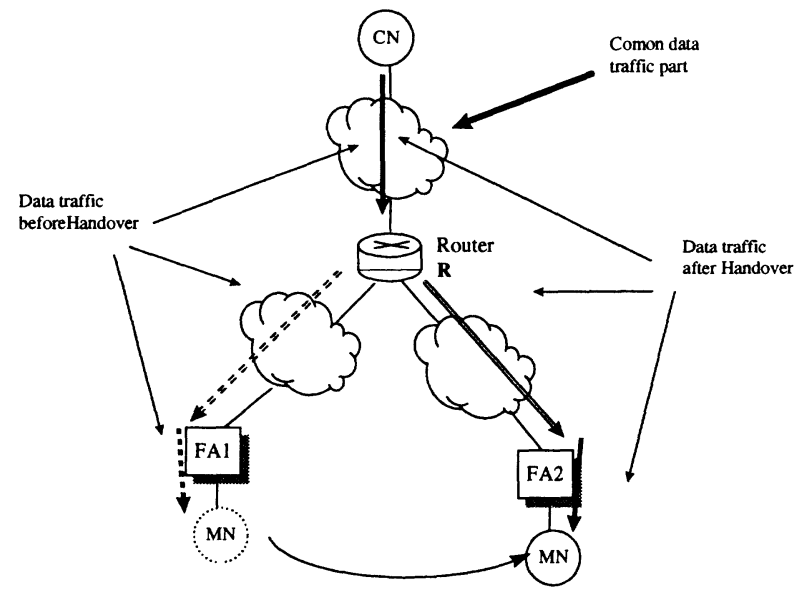

Figure 6: The handover procedure in combination with Mobile IPv6 and RSVP

The above procedures experience the following problems:

- Steps 2-5 are performed based on the "best effort" QoS, which may slow down and deteriorate the overall handover procedure. Additionally, the initiation of the new resource reservation at step 4 is not triggered by the reception of the binding update message at step 3 but only after the expiration of the "refresh timeout" period at the CN. This implies that in the meantime, some datagrams belonging to a flow from $\mathrm{CN}$ to $\mathrm{MN}$ may be treated by the routers as "best effort". This problem occurs due to the fact that the delivery of both the binding updates messages (they are sent by MN to both HA and the CN) and the RSVP messages (interchanged between the $\mathrm{CN}$ and the $\mathrm{MN}$ during the new resource reservation procedure) use the standard "best effort" IP service. Although in general, the delay of this procedure may not be considerable, its overhead is rather significant for the effective operation of the handover. Solutions such as the one used in connection oriented protocols (e.g. ATM) where a 
predefined channel is reserved for signaling [1], [18], can not be employed due the connectionless nature of the IP. We propose a more suitable approach, which must mark/tag the appropriate IP packets (note that both Mobile IP binding updates and RSVP messages are transported within IP datagrams) as time sensitive in order to obtain special care in the routers. This can be achieved by using the Traffic Class field in the header of the IPv6 datagram (or the TOS field in an IPv4 datagram) as described also in the differentiated services approach [5]. This requires interaction between the RSVP and IPv6 modules so as the IPv6 module will mark the RSVP messages correctly.

- The initiation of the new resource reservation procedure. After the initial resource reservation along the path to the $\mathrm{MN}$ has been set up, the $\mathrm{CN}$ will continue to transmit PATH refresh messages at regular intervals, since this is required by the RSVP's "soft state" operation. Then a binding update arrives from the $\mathrm{MN}$, informing $\mathrm{CN}$ of its current coa (coa2 after the handover). When the "refresh timeout" period expires at $\mathrm{CN}$, a PATH message is transmitted towards MN's new location (coa2) which establishes resource reservation along the new path. The default average period between transmissions of RSVP messages is 30 seconds [6], [7]. This period is quite long for the fast handover process. In the meantime, IP datagrams will be delivered to the MN using "best effort" service. We propose the following solutions to solve this problem:

$\checkmark$ Reduction of the RSVP retransmission period. The shorter this period is, the faster the response of the RSVP module at the CN about the change of the coa of the MN. However, the disadvantage of this approach is the increase of signaling overhead in the network, due to the fact that PATH messages are sent more frequently.

$\checkmark$ Another solution considers the triggering of the RSVP module when the binding update message arrives at the $\mathrm{CN}$. This arrival subsequently triggers the transmission of the PATH message immediately. However, this method requires modification of RSVP operation at the $\mathrm{CN}$, so that $\mathrm{CN}$ is informed of the arrival of the binding update message.

- Resource Reservation along the common path. Resource reservation will be made along the entire path from the $\mathrm{CN}$ to the MN. However, this may not be necessary for the common part between the old and new route (i.e. from $\mathrm{CN}$ to router $\mathrm{R}$ ). This occurs because data flow classification at the routers is based on MN's coa. This address changes after the handover has been performed (coa1 and coa2 the are the addresses before and after handover respectively). Although QoS reservation exists in the routers 
comprising the common path (from $\mathrm{CN}$ to $\mathrm{R}$ ), the data flow will not be identified as the one obtaining the reserved QoS. Data flow classification is based on the session spec (the triple destination address, protocol id and optional destination port) and sender id (source address, source port). MN's coa is employed to identify in the routers the IP datagrams requesting a certain QoS. When handover is triggered, the MN's coa changes and as an effect a new resource reservation must be made along the path although the appropriate resources have already been reserved in the common part from the $\mathrm{CN}$ to router R. This is illustrated in Figure 6. QoS reservation at routers is based on MN's ha not its coa. However, data traffic carries MN's coa as the destination address. Although QoS reservations may exist at the routers (based on MN's ha), IP datagrams (including MN's coa) will not be identified as the flow to obtain the reserved QoS. Therefore, we propose the following solutions:

$\checkmark$ Binding caches are employed at the routers. Routers record the bindings of the MNs and use this binding in order to route the RSVP messages towards the MNs. When a MN moves to a new $\mathrm{AP}$, a binding message is sent from the $\mathrm{MN}$ to the $\mathrm{CN}$. This message is also used by the routers to perform binding update and renew their cache with the new MN's coa (coa2). Thus the arriving packets coa2 will be identified as the ones which must receive the reserved resources in the routers along the common path (from $\mathrm{CN}$ to $\mathrm{R}$ ) where the reservation has been employed by using coal. However, the main disadvantage of this approach regards scalability due the extra memory requirements at the expense of resolving the handover issue.

$\checkmark$ Reservations are made at routers by using both MN's addresses (ha and coa). This approach alleviates the handover problem. This is due to the fact that when RSVP PATH messages have been processed along the entire path, the RSVP module has been updated using MN's coa2. However, in this case QoS reservation still has to be reestablished along the entire path. Additionally, the longer the path is, the longer the delay to update the RSVP module with the new MN coa. This approach may also lead to low throughput when the MN's movement is high.

- The soft state of RSVP does not require canceling the reservations in the old path (before handover) since this occurs when the relevant timers expire. The RSVP "soft state" approach defines that the RSVP states created at routers, are deleted if no matching refresh message arrives before the expiration of a "cleanup timeout" interval at the router. However the actual soft state timers are too long (around 30 secs) for fast 
handover purposes, which may lead to unnecessary resource reservation thus wasting the network's resources. This problem can be resolved by reducing the soft state timers at the expense of extra overhead in the RSVP messages. Another approach regards the employment of RSVP Teardown messages which remove path or reservation state immediately. There are two types of RSVP teardown message called PathTear and ResvTear [4], [6], [7], [19]. A PathTear message travels towards all receivers downstream from the point of initiation and deletes path state, as well as all dependent reservation state. A ResvTear message deletes reservation state and travels towards all senders upstream from the point of initiation. A PathTear (ResvTear) message may be visualised as a reversed-sense Path message (Resv message, respectively). Once it is initiated, a teardown request must be forwarded hop-by-hop without delay. A teardown message deletes the specified state in the received node. Based on this option, the $\mathrm{CN}$ can initiate a PathTear message towards FA1 immediately after receiving the binding update message from the $M N$. Then a new resource reservation along the path may be initiated. It must be emphasized that in this way the reservation along the common part of the path (from $\mathrm{CN}$ to $\mathrm{R}$ ) is torn down and a new one is re-created. The most efficient approach is to allow router $\mathrm{R}$ initiating the PathTear message towards the FA1 and subsequently the Path message is sent towards the MN. However but this approach lacks implementation simplicity.

\section{CONCLUSIONS}

This paper has addressed the problem of routing and handover of RSVP messages within mobile hosts. It has been demonstrated that RSVP suffers from serious problems of operation when combined with Mobile IP, due to fact that it has been designed for users attached to fixed network points. The problems of interworking of RSVP and Mobile IP is of great importance in order to provide QoS guarantees in mobile hosts. The issues of routing and handover associated with RSVP have been addressed when both Mobile IPv4 and IPv6 are employed and possible solutions have been proposed.

\section{REFERENCES}

[1] A. Acharya et al, "Mobility Management in Wireless ATM Networks", IEEE Communications Magazine, pp. 20-30, Nov. 1997.

[2] B. Balachandran et al, "Active Filters: Delivering Scalable Media to Mobile Devices", Proc. $7^{\text {th }}$ Int. Workshop on Network and Operating System Support for Digital Audio and Video, May, 1997. 
[3] B. Bardinath et al, "Ipv6+Mobile-IP+MRSVP = Internet Cellular Phone", Fifth IFIP Workshop on QoS, May 1997.

[4] B. Barzilai et al, "Design and Implementation of an RSVP-Based Quality of Service Architecture for an Integrated Services Internet", IEEE J. Selected Areas on Comm., pp. 397-413, April 1998.

[5] Y. Bernet et al, "A Framework for Differentiated Services", IETF, Work in progress, May 1998.

[6] R. Braden et al, "RSVP- Version 1: Functional Specifications", RFC-2205, Sept. 1997.

[7] R. Braden et al, "RSVP- Version 1:Message Processing Rules", RFC-2209, Sept. 1997.

[8] A. Campbell, "QOS-Aware Middleware for Mobile Multimedia Communications", J. on Multimedia Tools and Applications, Kluwer Academic Publishers, pp. 67-82, July 1998.

[9] J. Gomez and A. Campbell, "Delivering Adaptive Flows to Mobile Hosts over the Internet", Advance Project Report, Columbia University, January 1997.

[10] S. Hadjiefthymiades et al, "Mobility Management in an IP-based Wireless ATM Network“, ACTS Mobile Summit, Rhodos, Greece, June 1998.

[11] IEEE Internet Computing Magazine, January 1998.

[12] D. Johnson and C. Perkins, "Mobility support in IPv.6", IETF, Internet Draft, Work in progress, November 1996.

[13] A. Kaloxylos et al, "Mobility Management and Control Protocols for Wireless ATM Networks", IEEE Network Magazine, pp. 19-27, July/August 1998.

[14] C. Perkins and D. Johnson, "Mobility support in IPv6", ACM Mobicom, November 1996.

[15] C. Perkins, "IP Mobility Support", RFC-2002, October 1996.

[16] C. Perkins, Mobile IP: Design Principles and Practices, Addison-Wesley, 1998.

[17] A. Talukdar, "MRSVP: A Reservation Protocol for an Integrated Services Packet Network with Mobile Hosts", DCS-TR-337, Rutgers University, 1997.

[18] A. Terzis et al, "RSVP Operation over IP tunnels", IETF, Internet Draft, Work in progress, August 1998.

[19] L. Zhang et al, "RSVP: A new resource reservation protocol", IEEE Network Magazine, pp. 8-18, Sept. 1993. 